\title{
Enfermeiros X Atendimento ao paciente em tentativa de auto-extermínio
}

\author{
Nurses X Patient service in self-extermination attempt \\ Servicio de enfermeras X Paciente en intento de auto exterminación
}

Recebido: 31/07/2021 | Revisado: 07/08/2021 | Aceito: 12/08/2021 | Publicado: 15/08/2021

Laila Silva Divino

ORCID: https://orcid.org/0000-0001-9869-6656 Universidade Vale do Rio Verde, Brasil E-mail: lailadivino@outlook.com

Ranile Santos Silva

ORCID: https://orcid.org/0000-0002-5844-4224 Universidade Vale do Rio Verde, Brasil E-mail: ranilesantos@yahoo.com.br

Alessandra Mara Oliveira Dzivielevski

ORCID: https://orcid.org/0000-0003-2157-5631 Universidade Vale do Rio Verde, Brasil E-mail: aledzi2@yahoo.com.br

João Paulo Soares Fonseca

ORCID: https://orcid.org/0000-0003-4886-1718 Universidade Vale do Rio Verde, Brasil E-mail: joao.fonseca@unincor.edu.br

Nielly Andrade Carvalho Ribeiro

ORCID: https://orcid.org/0000-0002-8399-0657 Universidade Vale do Rio Verde, Brasil E-mail: niellyacr@gmail.com

Daniel Neves Santos

ORCID: https://orcid.org/0000-0002-5204-6110 Polícia Civil, Brasil

E-mail: ortopediadns@gmail.com

Elaine Aparecida Rocha Domingues ORCID: https://orcid.org/0000-0002-7589-2344 Universidade Vale do Rio Verde, Brasil E-mail: elainerocha.contato@gmail.com

Thuanny Fernandes Brito Noguchi ORCID: https://orcid.org/0000-0003-0584-212X Universidade Vale do Rio Verde, Brasil E-mail: enf.thuanny@gmail.com

Kerolayne Lemes Feliciano

ORCID: https://orcid.org/0000-0002-1761-1073 Universidade Vale do Rio Verde, Brasil E-mail: kerolayne.lemes@hotmail.com

\begin{abstract}
Resumo
Os objetivos deste trabalho foram conhecer os sentimentos dos enfermeiros diante do atendimento a pacientes em tentativa de autoextermínio, em conjunto descrever a atuação do enfermeiro no atendimento, verificar as possibilidades de que estes sentimentos possam causar algum tipo de distúrbio psicológico nos enfermeiros e compreender a realidade da rotina do enfermeiro frente ao primeiro atendimento na urgência e emergência a pacientes em tentativa de autoextermínio. Para tal estudo ser realizado, houve a participação de cinco enfermeiros que atuam em plantões no serviço de urgência e emergência (Pronto Socorro), por meio da análise do Discurso do Sujeito Coletivo (DSC), logo responderam questões que facilitou o entendimento dos fatos abordados. Em concordância com os resultados obtidos na pesquisa, podemos observar que o atendimento de Enfermagem na urgência e emergência é de extrema relevância para a diminuição de óbitos e tentativas de autoextermínio. O acolhimento realizado de maneira adequada facilita a coleta de informações, logo facilita o cuidado para com este paciente. Em concordância com os resultados obtidos na pesquisa, podemos observar que $o$ atendimento de Enfermagem na urgência e emergência é de extrema relevância para a diminuição de óbitos e tentativas de autoextermínio. $\mathrm{O}$ acolhimento realizado de maneira adequada facilita a coleta de informações, logo facilita o cuidado para com este paciente.
\end{abstract}

Palavras-chave: Suicídio; Enfermeiros; Assistência.

\section{Abstract}

The objectives of this study were to know the feelings of nurses regarding the care of patients in an attempt of selfextermination, together to describe the role of nurses in care, to verify the possibilities that these feelings may cause some kind of psychological disorder in nurses and to understand the reality of the nurse's routine in the first assistance 
in urgency and emergency to patients in self-extermination attempt. For this study to be carried out, there was the participation of five nurses who work on shifts in the urgency and emergency service (Emergency Room), through the analysis of the Collective Subject Discourse (CSD), then answered questions that facilitated the understanding of the facts discussed. In agreement with the results obtained in the research, we can observe that nursing care in urgent and emergency care is extremely important to reduce deaths and self-extermination attempts. Appropriate reception facilitates the collection of information, thus facilitating care for this patient. In agreement with the results obtained in the research, we can observe that nursing care in urgent and emergency care is extremely important to reduce deaths and self-extermination attempts. Appropriate reception facilitates the collection of information, thus facilitating care for this patient.

Keywords: Suicide; Nurses; Assistance.

\section{Resumen}

Los objetivos de este estudio fueron conocer los sentimientos de los enfermeros respecto al cuidado de los pacientes en un intento de autoexterminamiento, junto con describir el rol del enfermero en el cuidado, para verificar las posibilidades de que estos sentimientos puedan ocasionar algún tipo de trastorno psicológico en enfermeras y comprender la realidad de la rutina de la enfermera en la primera asistencia en urgencia y emergencia a pacientes en intento de autoexterminismo. Para la realización de este estudio se contó con la participación de cinco enfermeros que laboran en turnos en el servicio de urgencia y emergencia (Urgencias), mediante el análisis del Discurso Colectivo del Sujeto (CSD), luego respondieron preguntas que facilitaron la comprensión los hechos discutidos. De acuerdo con los resultados obtenidos en la investigación, podemos observar que la atención de enfermería en la atención de urgencia y emergencia es de suma importancia para reducir las muertes y los intentos de autoexterminación. La recepción adecuada facilita la recogida de información, facilitando así el cuidado de este paciente. De acuerdo con los resultados obtenidos en la investigación, podemos observar que la atención de enfermería en la atención de urgencia y emergencia es de suma importancia para reducir las muertes y los intentos de autoexterminación. La recepción adecuada facilita la recogida de información, facilitando así el cuidado de este paciente.

Palabras clave: Suicidio; Enfermeras; Asistencia.

\section{Introdução}

O cuidado de enfermagem realizado a pessoas atendidas na urgência/emergência por tentativa de autoextermínio é de extrema relevância, pois, o número de mortes por suicídio cresce a cada ano, porém as pessoas mais tentam o suicídio do que consumam o fato (Santos, Hildebrandt, Kinalski, Fukes, \& Leite, 2019). Diante desta informação, identificar a visão do profissional de enfermagem frente ao paciente em tentativa de autoextermínio nos possibilita descobrir como é para o enfermeiro tratar um paciente que teoricamente não deseja viver e é fundamental para prevenir futuros comportamentos suicida.

Segundo Silva e Marcolan (2019), a taxa de mortalidade por suicídio no Brasil, de 1996 a 2016, aumentou de 4,29 para 5,55, sendo a região Sul a de maior prevalência. Destaca-se nesse estudo que a maior parte das vítimas foram homens correspondendo a 79,04\% dos óbitos por suicídio. Ademais, o maior número concentrou-se nos adultos jovens, de 20 a 29 anos. Entender o que esses dados representam pode contribuir com o reconhecimento da magnitude do problema e com a elaboração de estratégias direcionadas.

O conceito saúde que hoje envolve a normalidade do funcionamento do corpo humano, o modo como a pessoa se enxerga em meio à sociedade, e a saúde mental que traz o equilíbrio emocional de cada ser levam a uma nova visão, que além de saudável fisicamente, há o lado humanizado que permite a melhor qualidade de vida possível. Por isso diante de tantas formas de óbito, o autoextermínio tem sido a menos aceita, pois o fato de um ser tomar a decisão de romper a própria vida gera um estado de comoção, de revolta, de não compreensão, enfim um misto de sentimentos que não podem ser entendidos por si só (Bezerra \& Sorpreso, 2016).

O óbito por autoextermínio tem tido um acréscimo muito grande no decorrer dos últimos anos considerando estatísticas do mundo inteiro, é um agravante que deveria estar no centro de problemáticas a ser discutido pela saúde pública, pois ela é a porta de entrada do Sistema Único de Saúde (SUS), ou seja, a organização do sistema começa no serviço primário através da prevenção, o fato de identificar pessoas com características de distúrbios mentais faz com que se esteja um passo à frente, 
trabalhando a prevenção, podendo diminuir assim as taxas de morbimortalidade por suicídio (Silva, Carvalho, Magalhães, \& Carvalho Jr, 2018).

Porém o sistema não consegue englobar e cuidar de todas estas pessoas que precisam de acompanhamento específico, devido ao fato de que nem sempre o paciente procura por ajuda ou mostra sinais relacionados a autoextermínio, com isso as unidades de urgência e emergência vem recebendo várias ocorrências relacionadas ao fato, tendo assim que estar preparadas para priorizar condições que ameaçam a vida. Além disso, a equipe de Atendimento Pré-Hospitalar (APH) deve ter em mente as intervenções necessárias a serem realizadas com o paciente durante o transporte, priorizando estabilizar o quadro clínico da vítima (Silva \& Kohlrausch, 2016).

A classe enfermagem ainda nos dias atuais se encontra em processo de crescimento profissional, de identificação e valorização perante a sociedade, logo, a postura positiva de cada profissional diante de situações inusitadas, causa cada vez mais estruturação e confiança no trabalho executado (Maria, Quadros, \& Grassi, 2012).

Assim como no serviço de urgência e emergência onde o profissional de enfermagem tem sua atuação de importância com este paciente no primeiro atendimento, em outros âmbitos ele pode adquirir a percepção de pessoas que apresentam sinais e características de autoextermínio, logo se pode prevenir o suicídio. Diante disto a Estratégia de Saúde da Família (ESF) é um dos recursos utilizados para identificar pacientes propensos a distúrbios mentais, podendo ser acompanhados, visto que se necessário há o auxílio do Centro de Atenção Psicossocial (CAPS), que proporciona o acolhimento do paciente e dos familiares que sofrem tanto quanto na circunstância (Santos et al., 2017).

No contexto do suicídio, os profissionais de enfermagem atuam na prevenção, promoção e tratamento dos pacientes (Coelho et al., 2021). Por isso o cuidado de enfermagem deve ser priorizado e valorizado, pois este lida diretamente com as ocorrências e contribui com agilidade na identificação desses pacientes, evitando assim novas tentativas de autoextermínio. Logo o enfermeiro deve ser qualificado para tais atendimentos e ser fonte principal de equilíbrio emocional diante dos fatos (Reisdorfer et al., 2015).

A rotina da área hospitalar principalmente na ala de Pronto Socorro requer muita atenção e conhecimento técnico, o que gera uma pressão e sobrecarga natural no profissional de enfermagem, logo em situações em que o enfermeiro realiza o atendimento de pacientes em tentativa de autoextermínio a responsabilidade se expande, tendo assim seus medos, frustrações e preocupações expostas (Burigo, Fagundes, Medeiros, Losso, \& Correa et al., 2015).

Dentre estes atendimentos distintos um do outro, o profissional pode ocasionar um risco potencial negativo relacionado ao seu trabalho, causando danos a si mesmo e a terceiros, ou seja, é preciso que o ambiente hospitalar seja benéfico e acolhedor diante de situações de tanta fragilidade (Pimenta \& Barros, 2019).

É de extrema importância compreender os sentimentos tanto do paciente quanto do profissional de enfermagem, colocando em ênfase o trabalho do enfermeiro nestas ocorrências em especifico, pois, a capacitação técnica e emocional deste possibilita uma conduta de excelência no primeiro atendimento ao paciente em tentativa de autoextermínio (Liba et al., 2016).

Diante disto a equipe de enfermagem deve ter um emocional equilibrado, orientação adequada, protocolos que permitam autonomia para ajuda destes pacientes, logo esses pilares permitem a sensibilização para conceber um atendimento de qualidade tanto no cuidar em si quanto no compreender estes pacientes. Acima de tudo à unidade deve se preocupar em disponibilizar apoio psicológico 'humanizado para tais profissionais, evitando assim o desenvolvimento de distúrbios psicológicos nestes (Fontão, Rodrigues, Lino, Lino, \& Kempfer et al., 2018).

Os inúmeros desafios que o enfermeiro encontra durante os plantões de urgência e emergência tem trago à tona que uma capacitação qualificada para manejo de tais casos é de extrema importância para a prevenção do autoextermínio, tanto para os pacientes quanto para o profissional, levando em consideração que o enfermeiro que almeja realizar um atendimento de qualidade e se vê insatisfeito com seu próprio trabalho por falta de conhecimento e apoio, pode adquirir distúrbios psicológicos 
como depressão, frustração, medo, Síndrome de Burnout, sendo capaz assim da tentativa de suicídio ou realmente consumar o fato para fugir dos problemas (Aguiar, Ceretta, \& Soratto, 2015).

Dentre os distúrbios psicológicos citados acima, a Síndrome de Burnout é a que mais acomete os profissionais de saúde nos dias atuais, segundo o Ministério da Saúde (2017), "tal síndrome apresenta-se por sintomas de exaustão extrema, estresse e esgotamento físico resultante de situações de trabalho desgastante, que demandam muita competitividade ou responsabilidade". Logo associando a Síndrome de Burnout ao primeiro atendimento do paciente em tentativa de autoextermínio, torna-se claro a pressão do trabalho difícil a cumprir, levando a dúvida do profissional se ele é capaz de cumprir com seus objetivos de intervenção de forma eficaz. Essa insegurança não trabalhada através de conversas, ponto de distração, troca de opiniões com a equipe, protocolos, manejos, faz com que se adquira a depressão profunda diante da impotência ao realizar o atendimento (Jantsch, 2017).

Portanto, a Unidade deve proporcionar apoio total aos profissionais, tanto nos âmbitos técnicos, disponibilizando treinamentos específicos, palestras direcionadas ao assunto, quanto nos âmbitos emocionais, ficando sempre atentos aos primeiros sintomas que podem ser apresentados, como dores de cabeça frequente, fadiga, hipertensão arterial, dores musculares, alteração repentina de humor e o isolamento que é uma das principais características observadas. Logo diagnosticando precocemente tais sintomas, mais rápido podem-se tratar os profissionais (Alves, Santos, Oliveira, \& Yamaguchi, 2018).

Devido ao ritmo acelerado de uma instituição hospitalar, e a responsabilidade com o próximo, a síndrome que mais tem acometido o profissional da saúde é a Bornout, por isso a importância de manter a saúde física e mental destes profissionais, pois muitos se exaustam em jornadas duplas de trabalho, e muitas das vezes é neste momento que a fadiga prevalece, causando adoecimento (França, Martino, Aniceto, \& Silva, 2012).

Levando em consideração tais fatos esta pesquisa tem por objetivo conhecer os sentimentos dos enfermeiros diante do atendimento a pacientes em tentativa de autoextermínio, em conjunto descrever a atuação do enfermeiro no atendimento, verificar probabilidades de que estes sentimentos possam causar algum tipo de distúrbio psicológico nos enfermeiros e compreender a realidade da rotina do enfermeiro frente ao primeiro atendimento na urgência e emergência a pacientes em tentativa de autoextermínio.

\section{Metodologia}

O vigente estudo é do tipo exploratório, descritivo, utilizando o método de análise do Discurso do Sujeito Coletivo (DSC), este visa conhecer os sentimentos dos enfermeiros diante do atendimento a pacientes em tentativa de autoextermínio. Tal pesquisa é qualitativa, que traduz e expressa o sentido dos fatos do mundo social, trata-se de conter a distância que ocorre entre o entrevistador e o entrevistado (Minayo, 1994).

O estudo em questão foi realizado na unidade de Pronto Atendimento da Fundação Hospitalar São Sebastião, localizada na Rua Pedro Bonesio, 236, Centro, no município de Três Corações, Minas Gerais. O referido Hospital possui 5.000m de área construída, 126 leitos (dentre eles destinados para atendimento clínico e cirúrgico (adultos e pediátricos), Unidade de Terapia Intensiva (adulto, neonatal e pediátrico), maternidade e pronto atendimento. Tem um corpo clínico formado por médicos e enfermeiros altamente capacitados, com atendimento para várias especialidades. Atualmente conta com 36 profissionais de Enfermagem.

Foram selecionados cinco enfermeiros que atuam em plantões no serviço de urgência e emergência (Pronto Socorro) da Fundação Hospitalar São Sebastião, e que vivenciam o primeiro atendimento ao paciente em tentativa de autoextermínio. Nesta pesquisa foi aplicado um questionário, onde os enfermeiros tiveram a oportunidade de relatar seus sentimentos, e angustias relacionada ao trabalho realizado. 
A pesquisa foi realizada com aqueles profissionais que concordaram, assinando assim o Termo de Consentimento Livre e Esclarecido.

Critérios de elegibilidade: Enfermeiros que atuam no Pronto Socorro do Hospital São Sebastião; Que possuem pósgraduação em Urgência e Emergência;

Critérios de não elegibilidade: Enfermeiros que não atuam no Pronto Socorro do Hospital São Sebastião; Não aceitam participar do estudo.

Os participantes desta pesquisa possuem características em comum, cujos critérios para seleção foram determinados pelo objetivo do referido estudo.

A coleta de dados foi realizada pela própria pesquisadora por meio de uma entrevista, ocasião que foi aplicado e preenchido os instrumentos. Após a aprovação do projeto pelo Comitê de Ética e Pesquisa (CEP) nº: 38818620.7.0000.5158 da UninCor, solicitação à instituição para a realização da pesquisa e concordância em participar do estudo por meio da assinatura no Termo de Consentimento Livre e Esclarecido (TCLE).

Os resultados foram apresentados através do Discurso do Sujeito Coletivo, de acordo com a natureza deles dos mesmos, conforme demonstrado na Figura 1.

Figura 1 - Representação esquemática das etapas para apresentação dos resultados.

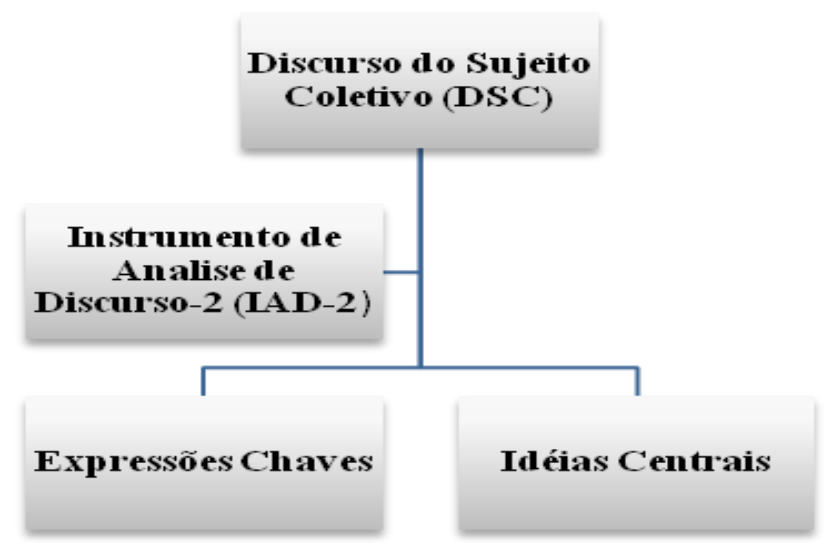

Fonte: Autores (2020).

Este estudo respeitou os preceitos éticos da resolução 466/12, do Conselho Nacional de Saúde (CNS), o qual se refere a estudos envolvendo seres humanos. Foram resguardadas a autonomia e privacidade dos participantes. Os mesmos foram esclarecidos quanto ao objetivo do estudo e assinaram o Termo de Consentimento Livre e Esclarecido. Número do parecer: 4000348030 .

\section{Resultados e Discussão}

\section{Descrição do perfil pessoal}

No início das entrevistas, realizou-se o levantamento de características pessoais dos enfermeiros entrevistados, bem como, idade, sexo e estado civil. Demarcando assim, o perfil de cada membro pesquisado. Os Gráficos 1, 2 e 3 demonstram a frequência relativa dos entrevistados quanto a idade, sexo e estado civil, respectivamente. 
Gráfico 1 - Frequência relativa dos entrevistados.

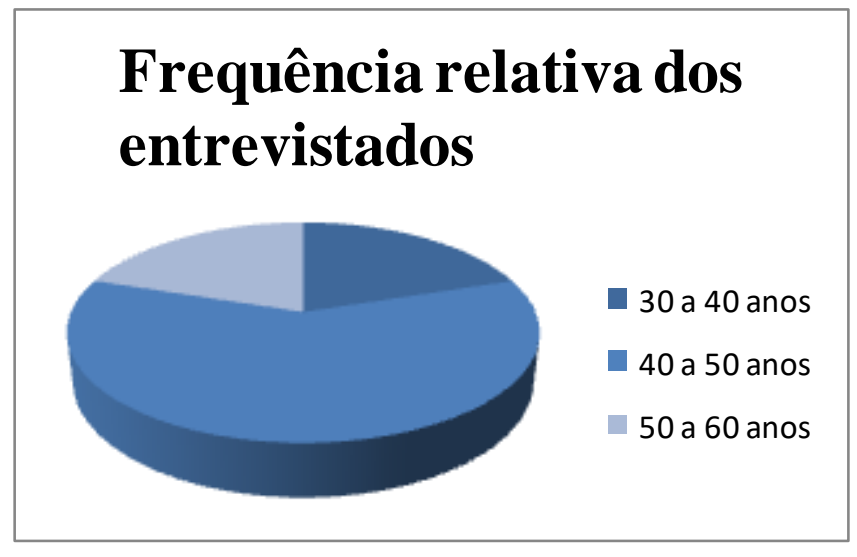

Fonte: Autores (2020).

Gráfico 2 - Sexo dos entrevistados.

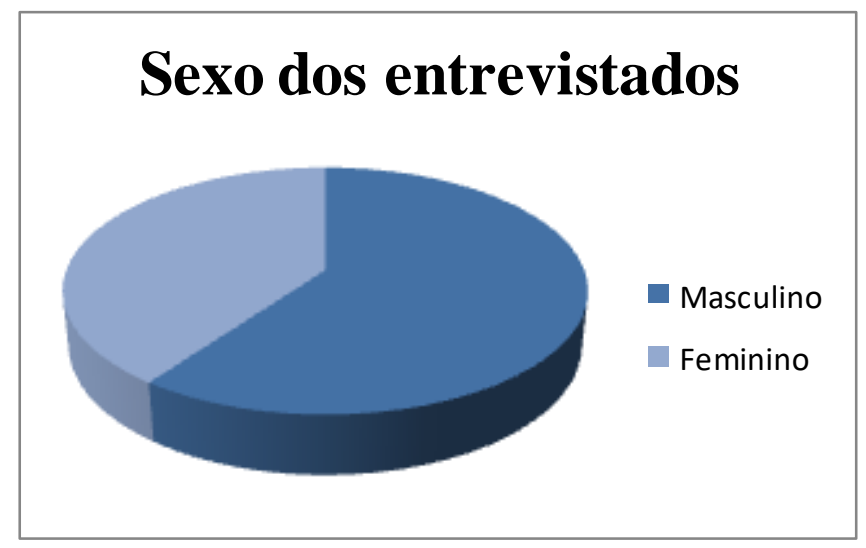

Fonte: Autores (2020).

Gráfico 3 - Estado civil dos entrevistados.

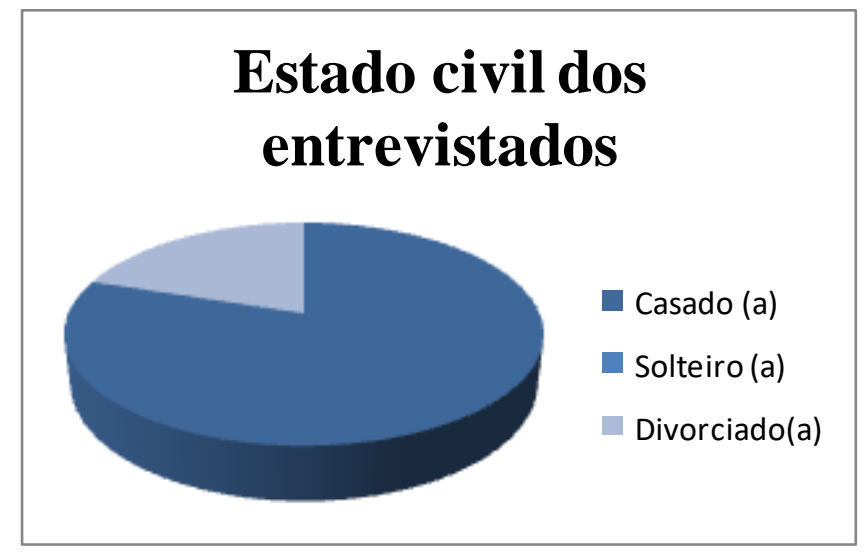

Fonte: Autores (2020).

Questão 1: Durante sua graduação em Enfermagem você recebeu orientações em disciplinas específicas direcionada à assistência de enfermagem na tentativa de autoextermínio? Elenque sobre seu ponto de vista. 
"Houve a matéria relacionada como um todo no psicossocial, o conhecimento adquirido na convivência também foi muito importante, pois vejo como um problema real" (D.S.C.1).

O profissional de enfermagem geralmente é o primeiro contato dos pacientes em tentativa de autoextermínio, diante disso sua avaliação e modo de agir para com este devem ser adequados ao momento, pois a falta de habilidade e conhecimento pode levar o afastamento deste, causando assim novas tentativas de suicídio (Fontão, Rodrigues, Lino, Lino, \& Kempfer, 2018).

Grande parte dos profissionais em Enfermagem se mostra capazes de cuidar de um paciente em tentativa de autoextermínio, porém esses cuidados foram adquiridos com a experiência adquirida durante os anos de profissão, pois poucos demonstram conhecimento desde formação acadêmica (Liba et al., 2016).

Questão 2: Elenque sobre seus sentimentos durante o primeiro atendimento ao paciente em tentativa de autoextermínio.

"O sentimento é uma mistura de tristeza, revolta, comoção, pena e que muda para o sentimento de raiva né, que as vezes o paciente precisa chamar atenção, por falta de acolhimento familiar. Temos sensibilidade e as vezes entendemos a situação do paciente" (D.S.C.2).

Na prestação de atendimento para estes indivíduos, é de extrema importância a qualidade emocional da equipe presente, devido ao fato da necessidade de deixar fatores externos fora do atendimento. Neste momento a humanização deve ir além, fazendo com que os sentimentos de compreensão e sensibilidade sejam aflorados (Braz, Ramos, \& Álvares, 2019).

É muito importante a maneira na qual o paciente em tentativa de autoextermínio é recebido em um pronto atendimento, pois estas atitudes sejam boas ou ruins vão influenciar no tratamento do mesmo. Logo em uma situação tão delicada os sentimentos da equipe de enfermagem são de grande relevância, aonde o medo, a raiva e a pena são os mais citados, e podem assim afastar o paciente (Kondo, Vilella, Borba, Paes, \& Maftum, 2011).

Questão 3: Na Unidade na qual você atua, existe o acompanhamento psicológico para os profissionais de enfermagem?

"Bom, não existe um acompanhamento. Existe assim, quando um ou outro profissional tem algum caso familiar, ou de tentativa de autoextermínio, ou de depressão, ou de qualquer outra coisa que envolva o colaborador da instituição, a equipe da assistente social junto ao psicólogo conversa com esse colaborador. Porém há certa resistência dos profissionais em procurar ajuda" (D.S.C.3).

A unidade hospitalar é um local que tem por objetivo cuidar, tratar, curar pessoas, e para que este trabalho seja bemsucedido a equipe também deve ser cuidada, tanto fisicamente quanto mentalmente. As queixas destes profissionais devem ser ouvidas, pois uma escuta qualificada gera outro ambiente de trabalho, e outra forma de enxergar os pacientes (Moretto et al., 2013).

A equipe de enfermagem deve estar bem preparada psicologicamente para fazer um bom atendimento a pacientes em tentativa de autoextermínio, pois o próprio enfermeiro tem sua saúde mental, que deve ser preservada, e deixada de lado no momento do atendimento, bem como mitos e crenças, julgamentos para com estes pacientes. Porém nem sempre há um atendimento especifico para os funcionários da instituição (Burigo, Fagundes, Medeiros, Losso, \& Correa, 2015).

Questão 4: Quais são os seus sentimentos em relação ao paciente que tenta o autoextermínio?

"Sentimento de tristeza, desolamento, decepção e até mesmo sensibilidade, cautela tolerância de entendimento a sua vulnerabilidade. Isso nos deixa chateado com os clientes, pois alguns estavam apenas querendo chamar atenção e outros queriam mesmo tirar a própria vida" (D.S.C.4).

A tentativa de autoextermínio é uma das causas mais frequentes recebidas em um pronto atendimento, logo o enfermeiro atribui um papel de grande importância com este paciente, pois com o atendimento correto se cria um vínculo com o mesmo, 
podendo assim evitar uma nova tentativa, ou seja, os sentimentos de raiva, medo, pena devem ser deixados de lado, para que haja um atendimento de excelência (Avanci, Furegato, Scatena, \& Pedrão, 2009).

Diante disto a equipe de enfermagem deve ter um emocional equilibrado, orientação adequada, protocolos que permitam autonomia para ajuda destes pacientes, logo esses pilares permitem a sensibilização para conceber um atendimento de qualidade tanto no cuidar em si quanto no compreender estes pacientes. Acima de tudo à unidade deve se preocupar em disponibilizar apoio psicológico humanizado para tais profissionais, evitando assim o desenvolvimento de distúrbios psicológicos nestes (Fontão el al., 2018).

Questão 5: Qual o procedimento quando, em um hospital ou unidade de saúde, é identificado um usuário que tentou autoextermínio?

"Depende da gravidade do ato. Nos casos mais graves, é passado de imediato para a assistente Social e psicóloga dentro do próprio hospital, onde ela faz o acompanhamento intra hospitalar, o enfermeiro faz a notificação, e são tomadas várias medidas para evitar qualquer outra tentativa enquanto estiver sob os cuidados da enfermagem. Após é encaminhado para PSF e CAPS" (D.S.C.5).

Segundo Freitas e Borges (2017), em pesquisa realizada sobre o atendimento às vítimas por tentativa de suicídio, apenas uma instituição tinha psicólogos na emergência e tinha como protocolo de atendimento o encaminhamento do paciente ao serviço de psicologia, quando possível.

A Rede de Atenção Psicossocial é de extrema importância na prevenção das tentativas de autoextermínios visto que vinculam os diversos serviços da rede de atenção à saúde que recebem pacientes que tentaram suicídio (Burigo et al., 2015).

Questão 6: Qual a sua maior dificuldade no atendimento com o paciente em tentativa de autoextermínio?

"A maior dificuldade é... creio que seja tentar colher informações do paciente, o atendimento especifico psicológico e fazer este paciente entender que a vida é bela, que vale a pena viver. De ordem emocional, enquanto acadêmicos nós não recebemos suporte teórico e técnico para nos direcionar quando tivermos que lidar com essa situação. Às vezes temos que tentar proteger este paciente de opiniões agressivas de outras pessoas" (D.S.C.6).

Grande dificuldade encontrada nas unidades de pronto atendimento é a hostilidade e negatividade de alguns profissionais para com estes pacientes, pois estas atitudes fazem com o que o paciente se afaste, não passe as informações necessárias para os cuidados, e aumenta o índice de novas tentativas, pois o transtorno mental se agrava (Vidal \& Gontijo, 2013). Segundo Santos (2015), o primeiro contato com o paciente vítima de tentativa de suicídio deve promover um vínculo de confiança e acolhimento.

\section{Considerações Finais}

Em concordância com os resultados obtidos na pesquisa, podemos observar que o atendimento de Enfermagem na urgência e emergência é de extrema relevância para a diminuição de óbitos e tentativas de autoextermínio. O acolhimento realizado de maneira adequada facilita a coleta de informações, logo facilita o cuidado para com este paciente.

Compreender a visão dos enfermeiros frente ao primeiro atendimento para o paciente em tentativa de autoextermínio possibilita a melhora na capacidade profissional, pois os enfermeiros com sua saúde mental cuidada conseguem ouvir melhor seu paciente, compreende-lo, chegando assim a melhor qualidade assistência.

Assim nos atentamos que a atenção básica, a atenção psicossocial e a atenção de urgência e emergência devem andar em conjunto, sempre pensando na saúde mental do paciente e do profissional, logo enquanto acadêmicos já se deve pensar na humanização, deixando de lado crenças, julgamentos e enxergar o paciente como um todo, que precisa de ajuda. 
Portanto um bom profissional é formado a partir de seus ideais, levando em consideração saber se aproximar deste paciente, ouvir o que ele tem a dizer sem pré-julgar, fazer os cuidados específicos em enfermagem, ter sensibilidade para com o próximo e tomar atitudes cabíveis para novas tentativas.

Sugere-se novos trabalhos a respeito da avaliação do estado psíquico dos profissionais enfermeiros que atendem pacientes vítimas de tentativas de suicídio.

\section{Referências}

Aguiar, E. B. P., Ceretta, L. B., \& Soratto, M. T. (2015). Os Desafios Enfrentados Pela Equipe De Enfermagem No Atendimento Ao Paciente Com Tentativa De Suicídio. RIES, 4(1), 68-82.

Alves, S. R., Santos, R. P., Oliveira, R. G., \& Yamaguchi, M. U. (2018). Serviços de saúde mental: percepção da enfermagem em relação à sobrecarga e condições de trabalho. J. res.: fundam. Care, 10(1), 25-29.

Avanci, R. C., Furegato, A. R. F., Scatena, M. C. M., \& Pedrão, L. J. (2009). Relação De Ajuda Enfermeiro-Paciente Pós-Tentativa De Suicídio. Revista Electrónica en Salud Mental, Alcohol y Drogas, 5(1), 1-15.

Bezerra, I. M. P. \& Sorpreso, I. C. E. (2016). Conceito de saúde e movimentos de promoção da saúde em busca da reorientação de práticas. J. Hum. Growth Dev., 26(1), 11-20.

Braz, T. C. O., Ramos, T. J. C. A., \& Álvares, A. C. M. (2019). Intervenção de enfermagem no âmbito de tentativas de pacientes autoextermínios em emergência hospitalar. Rev Inic Cient e Ext, 2(4), 241-6.

Burigo, E. B. F., Fagundes, M. J. D. M., Medeiros, I. S., Losso, A. R. S., \& Correa, S. M. (2015). A visão do enfermeiro no atendimento em tentativa de suicídio em um Pronto Socorro. RIES, 4(2), 26-39.

Coelho, A. K. R., Cunha, A. G., Silva, A. B. C., Cid, A. S., Castro, L. C. C., Lopes, B. C. M., Luz, B. N. M. N., Mendonça, J. S., Silva, L. P., Santos, L. J. C., Abreu, S. P. P., Franco, T. C., \& Silva, T. R. C. A. (2021). Práticas de enfermagem associadas às dinâmicas de prevenção ao suicídio: Um relato de experiência. Research, Society and Development, 10(4), e50310413819.

Fontão, M. C., Rodrigues, J., Lino, M. M., Lino, M. M., \& Kempfer, S. S. (2018). Cuidado de enfermagem às pessoas atendidas na emergência por tentativa de suicídio. Rev Bras Enferm, 71(suppl 5), 2329-35.

França, S. P. S., Martino, M. M. F., Aniceto, E. V. S., \& Silva, L. L. (2012). Preditores da Síndrome de Burnout em enfermeiros de serviços de urgência préhospitalar. Acta Paul Enferm. 25(1), 68-73.

Freitas, A. P. A. \& Borges, L. M. (2017). Do acolhimento ao encaminhamento: O atendimento às tentativas de suicídio nos contextos hospitalares. Estudos de Psicologia, 22(1), 50-60.

Jantsch, N. (2017). Síndrome de Burnout em profissionais de enfermagem: revisão integrativa. Artigo (Graduação) - Curso de Enfermagem, Universidade do Vale do Taquari - Univates.

Kondo, E. H., Vilella, J. C., Borba, L. O., Paes, M. R., \& Maftum, M. A. (2011). Abordagem da equipe de enfermagem ao usuário na emergência em saúde mental em um pronto atendimento. Rev Esc Enferm USP, 45(2), 501-7.

Liba, Y. H. A. O., Lemes, A. G., Oliveira, P. R., Nascimento, V. F., Fonseca, P. I. M. N., Volpato, R. J., Almeida, M. A. S. O., \& Cardoso, T. P. (2016). Percepções Dos Profissionais De Enfermagem Sobre O Paciente Pós-Tentativa De Suicídio. Journal Health NPEPS, 1(1), $109-21$.

Maria, M. A., Quadros, F. A. A., \& Grassi, M. F. O. (2012). Sistematização da assistência de enfermagem em serviços de urgência e emergência: viabilidade de implantação. Rev. Bras. Enferm., 65(2), 297-303.

Minayo, M. C. S. (1994). Pesquisa social: teoria, método e criatividade. Vozes.

Moretto, M. L. T. (2013). Cuidando de quem cuida": assistência psicológica ao trabalhador da saúde. Psicologia Hospitalar, 11(1), 52-65.

Pimenta, F. J. N. A. \& Barros, M. M. A. (2019). Ações e práticas de enfermagem frente ao paciente psiquiátrico atendido em um hospital de urgência e emergência de Porto Velho-RO. Revista Eletrônica Acervo em Saúde, 28, e1059.

Reisdorfer, N., Araujo, G. M., Hildebrandt, L. M., Gewehr, T. R., Nardino, J., \& Leite, M. T. (2015). Suicídio Na Voz De Profissionais De Enfermagem E Estratégias De Intervenção Diante Do Comportamento Suicida. Revista de Enfermagem da UFSM, 5(2), $295-304$.

Santos, E. G. O. (2015). O Cuidado do enfermeiro do setor de urgência e emergência ao paciente que tentou suicídio. Artigo Científico (Graduação em Enfermagem) - Faculdade de Ciências da Saúde do Trairi, Universidade Federal do Rio Grande do Norte, Santa Cruz.

Santos, L. F., Hildebrandt, L. M., Kinalski, S. S., Fukes, A. M. P., \& Leite, M. T. (2019). Atenção à pessoa com tentativa de suicídio em hospital geral: a voz de profissionais de enfermagem. Rev. Bras. Pesq. Saúde, 21(4), 27-37.

Santos, R. S., Albuquerque, M. C. S., Brêda, M. Z., Bastos, M. L. A., Silva, V. M. S., \& Tavares, N. V. S. (2017). A Atuação Do Enfermeiro Com A Pessoa Em Situação De Suicídio: Análise Reflexiva. Rev enferm UFPE, 11(2), 742-8. 
Research, Society and Development, v. 10, n. 10, e457101018945, 2021

(CC BY 4.0) | ISSN 2525-3409 | DOI: http://dx.doi.org/10.33448/rsd-v10i10.18945

Silva, D. A., \& Marcolan, J. F. (2019). Epidemiologia do suicídio no Brasil entre 1996 e 2016 e a política pública. Research, Society and Development, 9(2), e79922080.

Silva, N. K. N., Carvalho, C. M. S., Magalhães, J. M., Carvalho Junior, J. A. M., Sousa, B. V. S., \& Moreira, W. C. (2018). Ações do enfermeiro na atenção básica para prevenção do suicídio. SMAD Revista Eletrônica Saúde Mental Álcool E Drogas, 13(2), 71-77.

Silva, S. L. \& Kohlrausch, E. R. (2016). Atendimento pré-hospitalar ao indivíduo com comportamento suicida: uma revisão integrativa. SMAD Revista Eletrônica Saúde Mental Álcool E Drogas, 12(2), 108-115.

Vidal, C. E. L. \& Gontijo, E. D. (2013). Tentativas de suicídio e o acolhimento nos serviços de urgência: a percepção de quem tenta. Cad. Saúde Colet., 21(2): 108-14. 